\title{
Comportement du lupin blanc, Lupinus albus $L$, cv Lublanc, en sols calcaires. Seuils de tolérance à la chlorose.
}

\author{
C Duthion \\ Institut national de la recherche agronomique, centre de recherches de Dijon, station d'agronomie, \\ 17 rue Sully, BV 1540 Dijon Cedex 21034, France
}

(Reçu le 9 décembre 1991; accepté le 2 avril 1992)

\begin{abstract}
Résumé - Quarante quatre sols de textures relativement fines, contenant $0-46 \%$ de calcaire total, ont été prélevés dans différentes formations en Bourgogne et utilisés dans un essai de comportement du lupin blanc, cultivar Lublanc. Ce comportement a également été observé, dans un essai complémentaire, sur des sols de mélange obtenus par apport à l'un des sols de doses croissantes (0 à 15\%) d'un calcaire tamisé à 2 dimensions. La caractéristique des sols la plus explicative des résultats culturaux (symptômes de chlorose, teneur en chlorophylle, matière sèche produite) a été leur teneur en $\mathrm{CaCO}_{3}$ inférieure à $50 \mu \mathrm{m}$. Dans le premier essai, la corrélation entre les variables culturales et la teneur en $\mathrm{CaCO}_{3}$ total a été également forte. Les liaisons avec les teneurs en fer, extrait par 2 réactifs différents, avec les valeurs du $\mathrm{pH}$ eau et $\mathrm{CaCl}_{2}$, ont été lâches bien que significatives. Les indices de pouvoir chorosant se sont révélés de moins bons estimateurs du comportement du lupin blanc que les teneurs en $\mathrm{CaCO}_{3}$. Les teneurs critiques à retenir pour Lublanc sont $10 \%$ de $\mathrm{CaCO}_{3}$ inférieur à $50 \mu \mathrm{m}$, ou, dans les sols de textures fines au moins, $25 \%$ o de $\mathrm{CaCO}_{3}$ total.
\end{abstract}

lupin blanc $=$ Lupinus albus $L /$ chlorose $/$ calcaire $/$ fer $/$ teneur critique

Summary - Critical levels of $\mathrm{CaCO}_{3}$ in white lupin (Lupinus albus $\mathrm{L}$ ) cultivation. Fourty-four fine-textured soils, with total $\mathrm{CaCO}_{3}$ content from 0 to $46 \%$, were sampled from several areas in Burgundy. They were used to study white lupin behaviour in a greenhouse experiment with the cultivar Lublanc. This behaviour was also studied in an additional trial using soils obtained by mixing one of the 44 soils with increasing amounts (0-15\%) of a limestone sieved at 2 different sizes. Soil characteristic, which most explained the lupin results (chlorotic leaf symptoms, chlorophyll leaf content, total dry matter yield), was the content of $\mathrm{CaCO}_{3}$ with a size smaller than $50 \mu \mathrm{m}$. Total $\mathrm{CaCO}_{3}$ content was nearly as explicative for the natural soils. Lupin results on the contrary were poorly related to DTPA or $\left(\mathrm{COONH}_{4}\right)_{2}$ extractable $\mathrm{Fe}$ soil content and to water or $\mathrm{CaCl}_{2}$ soil $\mathrm{pH}$. IPC (capacity index for chlorosis) was not so good an indicator of white lupin behaviour as it was of $\mathrm{CaCO}_{3}$ content. From the results of these experiments, $10 \%$ CaCO $\mathrm{C}_{3}$ with a size smaller than $50 \mu \mathrm{m}$ is a critical level for cultivar Lublanc; $25 \%$ total $\mathrm{CaCO}_{3}$ can also be considered as critical in fine-textured soils.

white lupin = Lupinus albus $L /$ chlorosis / calcareous soil / iron / critical level

\section{INTRODUCTION}

Les espèces du genre Lupinus ont des exigences plus ou moins strictes vis-à-vis du pH des sols; à $\mathrm{pH}$ élevé, et particulièrement en présence de calcaire $\left(\mathrm{CaCO}_{3}\right)$, elles seraient incapables de prélever les quantités de fer qui leur sont nécessaires (Lopez-Bellido et Fuentes, 1986). Le lupin blanc ( $L$ albus $L$ ), l'espèce cultivée la plus productive sous nos conditions, se développe bien en sols modérément acides ou proches de la neutralité. Sa sensibilité connue au $\mathrm{CaCO}_{3}$ l'a fait utiliser de longue date comme plante test dans les études concernant la chlorose (Gouny et Mazoyer, 1953). Il est donc généralement admis de l'exclure des sols dont le $\mathrm{pH}$ est supérieur à 7. Pourtant, dans les nombreux essais que nous avons réalisés en sols neutres ou même légèrement calcaires, le lupin blanc a atteint fréquemment le potentiel de rendement qui lui est reconnu, à condition d'avoir été inoculé au semis par Rhizobium lupini (Amarger et Duthion, 1983; Duthion et al, 1987). Des symptômes chlorotiques ont été observés dans quelques uns de ces essais; ils correspondaient à des zones limitées où, à profondeur du sol moindre, la proximité du substrat cal- 
caire avait entraîné de faibles modifications des caractéristiques physico-chimiques du milieu racinaire.

Ainsi le lupin blanc est susceptible de croître sans problème apparent dans des situations dont il semblait devoir être exclu; mais les limites de sa tolérance à la présence de $\mathrm{CaCO}_{3}$ sont étroites. Préciser les sols sur lesquels cette culture, pratiquement encore au stade expérimental malgré ses fortes potentialités, pourrait être implantée et les caractéristiques qu'il serait utile de considérer pour aider à leur choix apparaît donc une nécessité. Dans ce but un essai a été conduit, utilisant des sols prélevés dans différentes formations de Bourgogne. Une seconde expérience, mettant en jeu un sol enrichi par des doses croissantes de $\mathrm{CaCO}_{3}$, le complète. Le comportement du cultivar de printemps le plus répandu a été observé sur chacun de ces supports; la correspondance entre ce comportement et plusieurs carctéristiques analytiques des sols a été recherchée.

\section{MATÉRIEL ET MÉTHODES}

\section{Essai 1}

\section{Les sols utilisés et leurs caractéristiques analytiques}

Les horizons supérieurs de 41 sols, ainsi que les horizons sous-jacents de 3 d'entre eux, ont été prélevés.
Ces sols avaient été retenus sur la base des minutes des prospections effectuées pour les cartes pédologiques au $1 / 100000$ de Dijon et Beaume. Deux exigences avaient dirigé leur choix, création d'une gamme assez étendue de teneur en $\mathrm{CaCO}_{3}$ total et appartenance à des classes texturales peu éloignées dans le diagramme du GEPPA (Groupe d'étude des problèmes de pédologie appliquée, France). Les substrats de ces sols sont divers : calcaires durs, marnes et calcaires marneux de différents étages, argiles micacées, éboulis, cailloutis ou grèves, alluvions ou colluvions calcaires. La numérotation attribuée à chaque horizon prélevé, dénommé sol dans ce qui suit, dépend uniquement de l'ordre alphabétique des communes d'origine.

La composition granulométrique, les teneurs en carbone, azote, phosphore, éléments échangeables, l'humidité équivalente de la fraction $<2 \mathrm{~mm}$ de chaque sol ont été déterminées selon les techniques analytiques courantes. Le $\mathrm{pH}$ a été mesuré en millieu eau et en milieu $\mathrm{CaCl}_{2}$ 0,01 M. La teneur en $\mathrm{CaCO}_{3}$ total a été mesurée par volumétrie du $\mathrm{CO}_{2}$ dégagé par attaque acide; sa répartition dans les différentes fractions granulométriques a été estimée par application de la même technique aux fractions séparées. Le $\mathrm{CaCO}_{3}$ actif a été déterminé sans broyage préalable. La quantité de fer disponible a été évaluée sous la forme facilement extractible (Juste et Pouget, 1972), mobilisable par l'oxalate d'ammonium $(\mathrm{COONH})_{2}$, et sous la forme extractible par l'acide diéthylènetriaminepentacétique (DTPA) selon la technique de Lindsay et Norvell (1978).

Dans le tableau I, sont données les valeurs extrêmes, la moyenne et la médiane de quelques unes des caractéristiques mesurées. Des corrélations significatives ont été mises en évidence entre certaines d'entre elles, positives entre teneurs en éléments fins ou matière organique et teneurs en fer, entre quantité

Tableau I. Caractéristiques des 44 sols de l'essai 1.

\begin{tabular}{|c|c|c|c|c|}
\hline & Minimum & Maximum & Moyenne & Médiane \\
\hline Particules $<20 \mu \mathrm{m} \%$ terre fine & 42 & 85 & 68 & 68 \\
\hline $\mathrm{pH} \mathrm{H} \mathrm{H}_{2} \mathrm{O}$ & 6,35 & 7,90 & 7,55 & 7,65 \\
\hline $\mathrm{pH} \mathrm{CaCl} 2$ & 6,00 & 7,45 & 7,10 & 7,15 \\
\hline $\mathrm{CaCO}_{3}$ total \% terre fine & 0,2 & 46,0 & 10,2 & 6,0 \\
\hline $\mathrm{CaCO}_{3}<20 \mu \mathrm{m} \%$ terre fine & 0,0 & 16,1 & 2,9 & 0,7 \\
\hline $\mathrm{CaCO}_{3}<50 \mu \mathrm{m} \%$ terre fine & 0,2 & 20,6 & 4,2 & 1,5 \\
\hline Fe extractible $\left(\mathrm{COONH}_{4}\right)_{2} \mathrm{ppm}$ & 52 & 1212 & 212 & 145 \\
\hline Fe extractible DTPA ppm & 19 & 146 & 40 & 30 \\
\hline IPC 1* & 0 & 45 & 6 & 0,5 \\
\hline IPC $2^{*}$ & 0 & 281 & 55 & 14 \\
\hline Graviers \% terre totale & 0 & 15 & 2,8 & 1,3 \\
\hline
\end{tabular}

* IPC $1=\left(\mathrm{CaCO}_{3}<50 \mu \mathrm{m}\right) \cdot 10^{4} /\left[\mathrm{Fe}-\left(\mathrm{COONH}_{4}\right)_{2}\right]^{2}$

* IPC $2=\left(\mathrm{CaCO}_{3}<50 \mu \mathrm{m}\right) \cdot 10^{4} /(\mathrm{Fe}-\mathrm{DTPA})^{2}$ 
de $\mathrm{CaCO}_{3}$ total et $\mathrm{pH}$, négatives entre $\mathrm{CaCO}_{3}$ et fer; mais ces liaisons sont lâches $(|r|<0,5)$. La corrélation entre la quantité totale de $\mathrm{CaCO}_{3}$ et la part de celui-ci qui est présente dans les fractions fines est plus étroite; elle n'explique néanmoins qu'environ $65 \%$ de la variance de cette dernière. La somme des quantités de $\mathrm{CaCO}_{3}$ déterminées dans les fractions granulométriques est voisine de la quantité totale mesurée dans le sol ( $r=0,998$, pente de la droite de régression $=$ 1,02). Les valeurs de $\mathrm{CaCO}_{3}$ actif obtenues pour la plupart des sols contenant moins de $5 \%$ de $\mathrm{CaCO}_{3}$ total ont peu de vraisemblance, sans doute en raison des sources d'erreurs évoquées dès l'origine par l'auteur de la technique (Drouineau, 1942); cette caractéristique n'a donc pas été utilisée pour rendre compte des résultats culturaux. Les quantités de fer extraites par les 2 réactifs sont assez étroitement corrélées ( $r=$ 0,858 ), bien que très différentes. Les IPC (Juste et Pouget, 1972) ont été calculés à partir de ces 2 valeurs et de la quantité de $\mathrm{CaCO}_{3}$ de taille inférieure à $50 \mu \mathrm{m}$.

\section{Cultures, observations et mesures}

Le cultivar Lublanc a été cultivé sous serre en pots contenant $5 \mathrm{~kg}$ séchés à l'air de chacun des 44 sols, tamisé à $2 \mathrm{~cm}$. Chaque pot a reçu une fertilisation préalable comportant $2,8 \mathrm{~g} \mathrm{~K}_{2} \mathrm{HPO}_{4}, 1,5 \mathrm{~g} \mathrm{MgSO}_{4}$, $7 \mathrm{H}_{2} \mathrm{O}, 100 \mathrm{mg} \mathrm{CuSO}, 5 \mathrm{H}_{2} \mathrm{O}$ et $\mathrm{MnSO}_{4}, \mathrm{H}_{2} \mathrm{O}, 10 \mathrm{mg}$ $\mathrm{ZnSO}_{4}, 7 \mathrm{H}_{2} \mathrm{O}, \mathrm{H}_{3} \mathrm{BO}_{3}$ et $\left(\mathrm{NH}_{4}\right)_{2} \mathrm{MO}_{2} \mathrm{O}_{7}$. Huit graines ont été mises en place par pot et ont été inoculées par une suspension de $R$ lupini; 5 plantules ont été conservées après éclaircissage au stade 2 feuilles étalées. Les apports d'eau ont été conduits de telle façon que la teneur en eau du sol ne dépasse pas environ $90 \%$ de l'humidité équivalente. Le dispositif en blocs comprenait 4 répétitions.

Des notations de symptômes de chlorose ont été effectuées en début de végétation, selon une échelle de 0 (absence de symptôme) à 5 (nécrose, dessèchement). Une note composée moyenne par pot, tenant compte des notations faites sur les $2^{\mathrm{e}}, 4^{\mathrm{e}}$ et $6^{\mathrm{e}}$ feuilles de la tige principale des 5 plantes, au moment de leur expansion maximale, a servi à rendre compte de l'intensité des symptômes.

La teneur moyenne en chlorophylle des feuilles a été déterminée au début de la floraison de l'axe principal. L'échantillon a été constitué de la $4^{e}$ feuille de la plante $1,6^{\mathrm{e}}$ feuille de la plante $2, \ldots, 12^{\mathrm{e}}$ feuille de la plante 5 d'un pot, les feuilles prélevées dans les pots des blocs I et II d'une part, III et IV d'autre part d'un même traitement étant réunies. Les pigments ont été extraits par l'acétone à $80 \%$. La chlorophylle a été mesurée par spectrophotométrie et sa teneur calculée à partir des formules de MacKinney (1941) citées par Holden (1965).

À maturité, les parties aériennes de toutes les plantes d'un pot ont été récoltées ensembles. Leurs différents éléments, tiges, gousses, graines, ont été séparés et pesés après séchage à $80^{\circ} \mathrm{C}$ pendant $48 \mathrm{~h}$.

\section{Essai 2}

\section{Les sols et leurs caractéristiques analytiques}

Les sols de cet essai ont été préparés par mélange dans une bétonnière du sol $n^{\circ} 10$ de l'essai $1(76 \%$ argile + limon fin, $\left.0,2 \% \mathrm{CaCO}_{3}\right)$ tamisé à $2 \mathrm{~mm}$, de sable quartzeux $\left(10 \%\right.$ en poids de la terre $\left.n^{\circ} 10\right)$ et d'un calcaire tendre oolithique provenant de 21Lamargelle. Broyé et tamisé à 2 dimensions, l'une comprise entre 1 et $2 \mathrm{~mm}$ et l'autre inférieure à 125 $\mu \mathrm{m}$, celui-ci a été ajouté en quantités telles que les teneurs en $\mathrm{CaCO}_{3}$ total soient voisines de $0,1,2,4,6$, 9,12 et $15 \%$.

Après maintien des sols à $18-20 \%$ d'humidité pendant 4 semaines, leur teneur en $\mathrm{CaCO}_{3}$ total et sa répartition dans les différentes fractions granulométriques, celle en $\mathrm{CaCO}_{3}$ actif et en fer facilement extractible $\left(\mathrm{COONH}_{4}\right)_{2}$ ont été déterminées selon les techniques précédemment décrites. La corrélation significative entre $\mathrm{CaCO}_{3}$ total et la part de celui-ci contenu dans les fractions fines rend compte de moins de $40 \%$ de la variance de cette dernière. En revanche, la teneur en fer facilement extractible, qui varie de 243 ppm dans le mélange sans adjonction de $\mathrm{CaCO}_{3}$ à $105 \mathrm{ppm}$ dans le mélange ayant reçu 15\% de $\mathrm{CaCO}_{3}<125 \mu \mathrm{m}$, est fortement corrélée à la teneur en $\mathrm{CaCO}_{3}$ total. Les réserves sur les valeurs du $\mathrm{CaCO}_{3}$ actif, déjà formulées pour certains des sols de l'essai 1 , ont conduit à ne pas le retenir non plus comme variable explicative dans le second essai.

\section{Cultures, observations et mesures}

Le cultivar Lublanc a été cultivé sous serre en pots contenant $2 \mathrm{~kg}$ (sur la base du poids séché à l'air) de l'un des mélanges, dans un dispositif en randomisation totale comportant 3 répétitions. Chaque pot a reçu $3 \mathrm{~g} \mathrm{~K}_{2} \mathrm{HPO}_{4}$ et $1,25 \mathrm{~g} \mathrm{MgSO}_{4}, 7 \mathrm{H}_{2} \mathrm{O}$. Six graines ont été mises en place et 4 plantules conservées après éclaircissage précoce. L'inoculation par $R$ Lupini a été réalisée au semis.

Des notations de symptômes de chlorose ont été faites en cours de végétation, comme dans l'essai 1. Les plantes de chaque pot ont été récoltées globalement au début floraison des axes apicaux de second ordre et pesées après séchage à $80^{\circ} \mathrm{C}$ pendant $48 \mathrm{~h}$.

\section{RÉSULTATS ET DISCUSSION}

\section{Comportement et croissance des plantes en fonction des caractéristiques des sols}

Dans l'essai 1, toute l'étendue de l'échelle de notation des symptômes sur feuilles a été utilisée. La note composée, qui a été retenue pour les 
comparaisons, a varié de 0 à 7,5 (moyenne 1,8; médiane 0,8). La teneur en chlorophylle totale des feuilles prélevées était comprise entre 1 et $2,3 \%$ de la matière fraîche (moyenne $1,8 \%$, médiane $1,9 \%$ ). La quantité de matière sèche (MS) récoltée par pot a varié de 8 à $128 \mathrm{~g}$ (moyenne $71 \mathrm{~g}$, médiane $68 \mathrm{~g}$ ), tous organes confondus. La distribution des valeurs de ces 3 variables n'a pas été influencée de façon apparente par la classe texturale des sols, leurs teneurs en matière organique ou acide phosphorique ou leur humidité équivalente, qui sont susceptibles d'après plusieurs auteurs cités par Juste et Pouget (1980) de modifier les manifestations de la chlorose ferrique.

Dans l'essai 2 toute la gamme des symptômes a également été observée et les notes composées se sont établies entre 0 et 5 (moyenne 0,9 ). La MS récoltée a varié de 11 à $37 \mathrm{~g} / \mathrm{pot}$ (moyenne $25 \mathrm{~g}$ ), la valeur supérieure correspondant au traitement sans apport de $\mathrm{CaCO}_{3}$.

Dans les tableaux II pour l'essai 1 et III pour l'essai 2 sont indiqués les coefficients de corrélation linéaire entre les variables mesurées sur plantes et les caractéristiques de sols a priori explicatives de leurs comportements. Toutes ces corrélations sont significatives au seuil $5 \%$ mais beaucoup sont peu étroites. D'autres types de régression ont été testés, qui n'améliorent pas les ajustements aux valeurs expérimentales.

Les teneurs en $\mathrm{CaCO}_{3}$ sont les caractéristiques des sols les plus explicatives des résultats de l'essai 1; la teneur en $\mathrm{CaCO}_{3}<50 \mu \mathrm{m}$ en rend un peu mieux compte que la teneur totale. Dans l'essai 2, le comportement des plantes a été très lié à la teneur des sols en $\mathrm{CaCO}_{3}<20$ ou $50 \mu \mathrm{m}$; la valeur assez peu explicative dans ce cas de $\mathrm{CaCO}_{3}$ total est à rapprocher de l'utilisation de 2 lots de tailles très différentes du même calcaire pour la préparation des sols. Dans les 2 essais, les effets négatifs de la présence de $\mathrm{CaCO}_{3}$ ont été plus marqués que ceux obtenus par Schinas et Rowell (1977). Opérant avec un génotype différent de Lublanc et des sols de textures fines mais diluées pour moitié de sable quartzeux, ces auteurs n'ont d'ailleurs noté de relation nette entre croissance de jeunes plantes et teneur en $\mathrm{CaCO}_{3}$ total de ces mélanges que pour des valeurs de celle-ci supérieure à 10\%; en-deça, la variabilité des productions pour des teneurs voisines, mêmes très basses, était forte. Le rôle majeur des fractions fines de $\mathrm{CaCO}_{3}$ dans l'induction de la chlorose, qui se dégage des 2 essais, est un fait depuis longtemps signalé (Drouineau, 1942).

Les effets observés sont peu expliqués par les teneurs en fer dans l'essai 1, davantage dans l'essai 2; pour ce dernier la variation de teneur a été induite dans un même milieu. En fait, toutes les concentrations mesurées apparaissent relativement élevées. La comparaison des sols de l'essai 1 avec ceux utilisés par Lindsay et Norvell (1978) dans la mise en évidence d'un niveau critique pour le sorgho le montre particulièrement. Pourtant, cette richesse en fer n'a pas évité tout symptôme de chlorose; les plus sévères, dans l'essai 1 , ont été observées sur le sol $n^{\circ} 20$ contenant $171 \mathrm{ppm} \mathrm{Fe}\left(\mathrm{COONH}_{4}\right)_{2}$. L'examen de ces résultats conduit à poser l'hypothèse que le comportement du lupin blanc en sol calcaire, contrairement à celui d'autres espèces, vigne, sorgho ou même soja, est davantage influencé par la quantité de calcaire présente que par la disponibilité en fer.

Tableau II. Coefficients de corrélation entre caractéristiques des sols (variables explicatives) et variables de comportement du lupin blanc (variables expliquées) dans l'essai $1: 42$ degrés de liberté.

\begin{tabular}{lccr}
$\begin{array}{l}\text { Caractéristiques } \\
\text { des terres }\end{array}$ & $\begin{array}{c}\text { Notation } \\
\text { symptômes }\end{array}$ & $\begin{array}{c}\text { Variables de comportement du lupin blanc } \\
\text { Teneur en } \\
\text { chlorophylle }\end{array}$ & $\begin{array}{c}\text { Matière sèch } \\
\text { récolte }\end{array}$ \\
\hline & & & $-0,78$ \\
$\mathrm{CaCO}_{3}$ total & 0,84 & $-0,49$ & $-0,67$ \\
$\mathrm{CaCO}_{3}<20 \mu \mathrm{m}$ & 0,82 & $-0,73$ & $-0,70$ \\
$\mathrm{CaCO}_{3}<50 \mu \mathrm{m}$ & 0,86 & $-0,76$ & 0,41 \\
$\mathrm{Fe}-\left(\mathrm{COONH}_{4}\right)_{2}$ & $-0,41$ & 0,31 & 0,36 \\
$\mathrm{Fe}-\mathrm{DTPA}$ & $-0,32$ & 0,29 & $-0,52$ \\
$\mathrm{IPC} 1$ & 0,73 & $-0,62$ & $-0,60$ \\
$\mathrm{IPC} 2$ & 0,80 & $-0,74$ & $-0,43$ \\
$\mathrm{pH} \mathrm{CaCl}$ & 0,35 & $-0,37$ &
\end{tabular}


Tableau III. Coefficients de corrélation entre caractéristiques des sols (variables explicatives) et variables de comportement du lupin blanc (variables expliquées) dans l'essai 2 : 13 degrés de liberté.

\begin{tabular}{lrc}
$\begin{array}{l}\text { Caractéristiques } \\
\text { des terres }\end{array}$ & $\begin{array}{c}\text { Notation } \\
\text { symptômes }\end{array}$ & $\begin{array}{c}\text { Matière sèche } \\
\text { récolte }\end{array}$ \\
\hline $\mathrm{CaCO}_{3}$ total & 0,64 & $-0,66$ \\
$\mathrm{CaCO}_{3}<20 \mu \mathrm{m}$ & 0,98 & $-0,88$ \\
$\mathrm{CaCO}_{3}<50 \mu \mathrm{m}$ & 0,98 & $-0,88$ \\
$\mathrm{Fe}-\left(\mathrm{COONH}_{4}\right)_{2}$ & $-0,75$ & 0,75 \\
$\mathrm{IPC} 1$ & 0,93 & $-0,76$ \\
\hline
\end{tabular}

Conséquemment, la valeur explicative de I'IPC, très forte dans le cas de la vigne, serait moindre pour le lupin blanc; celle des expressions voisines, qui ont été utilisées, a été en effet, dans les 2 essais, intermédiaire entre celles de $\mathrm{CaCO}_{3}<50 \mu \mathrm{m}$ et du fer.

La liaison entre les résultats culturaux de l'essai 1 et le $\mathrm{pH}$ des sols (non mesuré dans l'essai 2), a été faible, contrairement à ce qu'avaient noté Schinas et Rowell (1977) sur des sols de Grande-Bretragne; pour ces auteurs, $69 \%$ de la variabilité de la production de matière fraîche par le lupin blanc étaient associés aux variations du $\mathrm{pH} \mathrm{CaCl}_{2}$ des sols.

La réduction de croissance, lorsqu'elle a été forte, a entraîné une diminution de l'efficience de la MS élaborée à former des graines et de sa capacité ultérieure à les remplir. Ainsi, pour les 10 sols de l'essai 1 qui ont permis la croissance la plus importante, le rapport du nombre de graines récoltées à la $M S$ hors graines à la récolte a été en moyenne de 3,4 graines $/ g$ MS et le poids moyen d'une graine de $298 \mathrm{mg}$; pour les 10 sols de ce même essai sur lesquels a été observée la croissance la plus faible, ces grandeurs ont été respectivement de 2,6 graines/g MS et $205 \mathrm{mg}$. Cet effet ne peut cependant être considéré comme symptomatique de la présence de $\mathrm{CaCO}_{3}$ dans les sols.

\section{Seuils de tolérance à la présence de calcaire}

Les sols de l'essai 1 ont été rangés en 5 classes, faisant intervenir les 3 critères de comportement du lupin blanc dont il a été tenu compte (tableau IV). Le sol $n^{\circ} 10$, sur lequel des cultures au champ ont été effectuées plusieurs années successives avec des rendements proches du po-
Tableau IV. Les critères de classement des sols de l'essai 1 en 5 classes.

\begin{tabular}{lcc}
\hline $\begin{array}{c}\text { Symptômes } \\
\text { de chlorose }\end{array}$ & $\begin{array}{c}\text { Teneurs } \\
\text { en } \\
\text { chlorophylle }\end{array}$ & $\begin{array}{c}\text { Production MS } \\
\text { terre } n^{\circ} 10\end{array}$ \\
& normale & $90<$ \\
Classe A aucun & normale & $70<\leq 90$ \\
Classe B aucun & normale & $50<\leq 70$ \\
Classe C modérés & $<$ normale & $30<\leq 50$ \\
Classe D modérés à forts & $<$ normale & \\
Classe E forts & & \\
& & \\
\hline
\end{tabular}

tentiel de l'espèce (Duthion et al, 1987), a tenu lieu de témoin pour la production de MS. La teneur en chorophylle a été jugée normale quand elle est supérieure à $1,6 \%$ de la matière fraîche (Zelitch, 1971). Les sols des classes A et B ont été considérés comme pouvant convenir à la culture du lupin blanc. Les sols de l'essai 2 ont été simplement classés en sols convenant (MS $\geq 0,7$ MS témoin, absence de symptômes) ou ne convenant pas à la culture.

La teneur en $\mathrm{CaCO}_{3}<50 \mu \mathrm{m}$ paraît l'indicateur le plus pertinent pour apprécier les risques liés à la présence de calcaire. Selon les résultats de l'essai 1, le seuil de tolérance de Lublanc se situe à une teneur voisine de $10 \%$ on $\mathrm{CaCO}_{3}$ $<50 \mu \mathrm{m}$ (fig 1a). La prise en considération de cette valeur critique aurait permis de rejeter la totalité des sols des classes C, D et E et $90 \%$ des sols des classes $A$ et $B$ auraient été jugés propres à la culture. La conclusion est identique à partir de l'essai 2; le niveau du seuil de tolérance qui en est tiré, approximativement $7 \%$, est cependant légèrement inférieur au précédent, parce que les sols de mélange n'ont sans doute par encore atteint leur état d'équilibre, et qu'une plus grande imprécision existe dans ce cas, due à la gamme des teneurs réalisées. La concordance de ces données avec les résultats de Gouny et Mazoyer (1953) est remarquable. Ces auteurs ont étudié en effet un génotype, non identifié, de lupin blanc placé sur des milieux constitués d'un sable quartzeux auquel ont été apportés $0-1-2,5$ jusqu'à $20 \%$ de $\mathrm{CaCO}_{3}$ précipité, donc de petites dimensions. Les symptômes de chlorose, qu'il ont décelés, étaient à peine perceptibles et non accompagnés de réduction de croissance à $1 \%$ de $\mathrm{CaCO}_{3}$, mais déjà accentués à $2,5 \%$.

La teneur en $\mathrm{CaCO}_{3}$ total se montre un indicateur satisfaisant dans l'essai 1 , où avec un ni- 


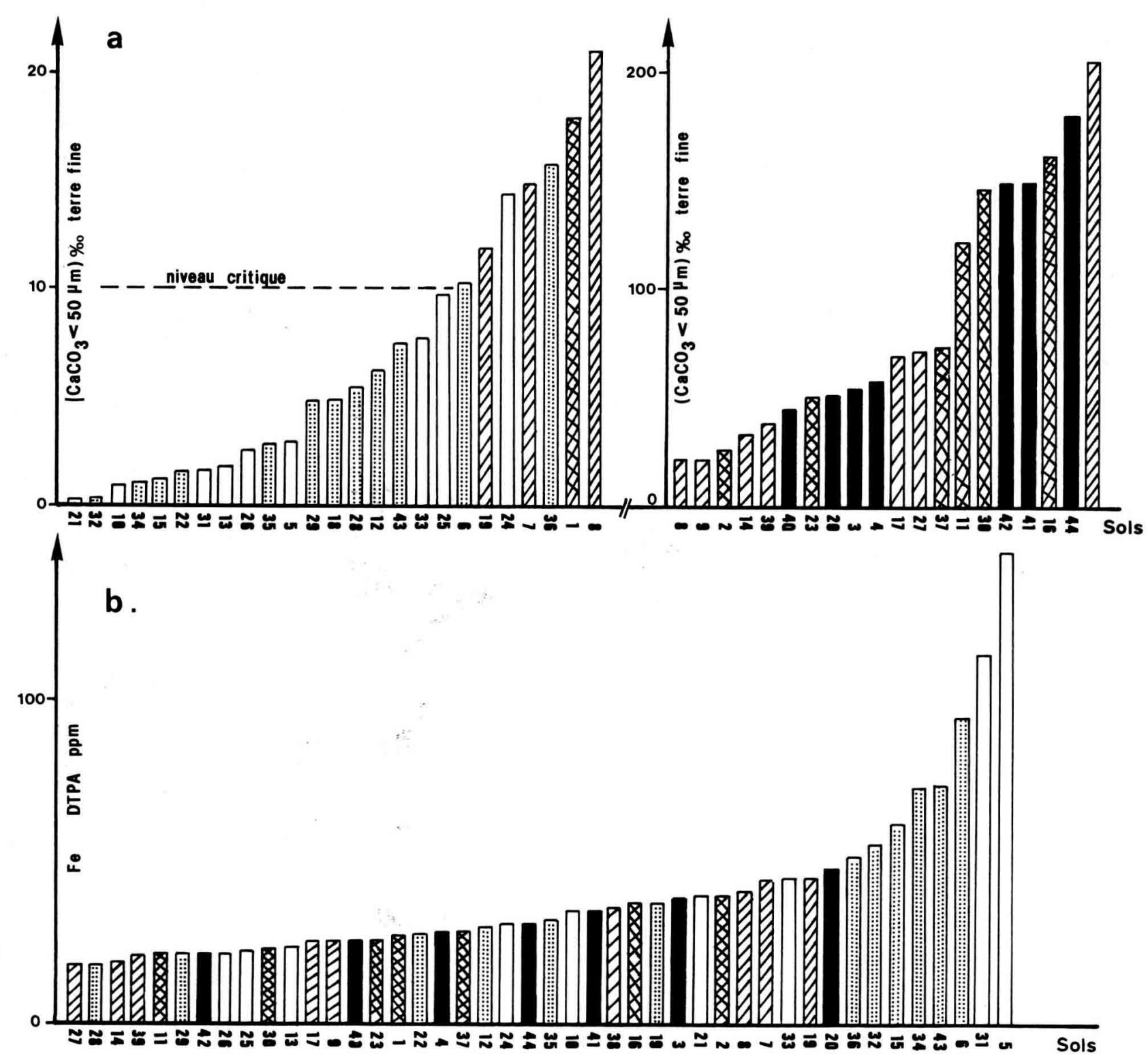

Fig 1. Les 5 classes des sols de l'essai 1 représentées en fonction des teneurs croissantes : a en $\mathrm{CaCO}_{3}$ de taille inférieure à $50 \mu \mathrm{m}$; $b$ en Fe extrait par DTPA; classe A : $\square$ classe B :

veau critique de $25 \%$ elle aurait conduit cependant à n'accepter que $75 \%$ des sols $\mathrm{A}$ et $\mathrm{B}$. En revanche dans l'essai 2 elle se révèle sans valeur prédictive, pour la raison invoquée dans le paragraphe précédent.

Les teneurs en fer, extrait par l'une ou l'autre technique, apparaissent de peu d'intérêt pour le diagnostic (fig 1b) et, dans cette perspective, ne sont pas très différentes du $\mathrm{pH}$ des terres. Sous ses formes modifiées par rapport à l'expression proposée par Juste et Pouget (1972), l'IPC aurait été trop ou insuffisamment discriminant; ainsi, I'utilisation des valeurs d'IPC1 et d'IPC2, permettant d'écarter tous les sols C, D, E de l'essai 1 conduirait à considérer comme impropres à porter du lupin blanc 50 et $30 \%$ des sols A et B.

\section{CONCLUSION}

Le comportement du lupin blanc sur l'ensemble des sols des 2 essais de l'étude est apparu en liaison assez étroite avec les teneurs en $\mathrm{CaCO}_{3}$ présent dans les fractions argile et limon. Dans les sols de l'essai 1, d'origines sans doute voisines et appartenant à des classes texturales proches, il a été également plutôt bien corrélé aux teneurs en $\mathrm{CaCO}_{3}$ total. Les autres caractéristiques des 
terres en ont été assez peu explicatives. Au-delà d'une teneur de $10 \%$ de la terre fine en $\mathrm{CaCO}_{3}<$ $50 \mu \mathrm{m}$, les risques d'apparition de symptômes de chlorose et d'effets négatifs sur la croissance sont importants. Dans des sols tels que ceux de l'essai 1, la teneur en $\mathrm{CaCO}_{3}$ total, avec un niveau critique de $25 \%$, pourrait aussi être utilisée comme élément de choix. II est possibie qu'en sols de texture beaucoup plus grossière, donc moins riches en fer, les indicateurs qui ont été écartés soient préférables aux teneurs en $\mathrm{CaCO}_{3}$ ou que d'autres teneurs critiques soient à retenir.

Les résultats concernent uniquement le cultivar Lublanc. Bien qu'aucune différence n'ait été notée en culture parmi les cultivars actuellement disponibles, la variabilité génotypique serait cependant à explorer.

\section{REMERCIEMENTS}

L'auteur remercie J Chrétien, du SESCPF, de lui avoir permis de consulter les relevés pédologiques qu'il a effectués dans la région Bourgogne et $V$ Durey, $\mathrm{J}$ Gonthier, $\mathrm{P}$ Mathey pour leur aide technique.

\section{RÉFÉRENCES}

Amarger N, Duthion C (1983) Effets de l'inoculation par Rhizobium et de la fertilisation azotée sur les rendements et la teneur en azote des graines de lupin (Lupinus albus, L mutabilis, L luteus). Agronomie 3, 995-1000

Drouineau G (1942) Dosage rapide du calcaire actif des sols. Nouvelles données sur la répartition et la nature des fractions calcaires. Ann Agron, 441-450

Duthion C, Amarger N, Mariotti A (1987) Accumulation potentielle de matière sèche et d'azote chez le lupin blanc de printemps (Lupinus albus L). Agronomie 7, 585-593

Gouny P, Mazoyer R (1953) Relation entre la nutrition minérale et les symptômes pathologiques dans la chlorose calcaire. Ann Agron 4, 561-597

Holden M (1965) Analytical methods. Chlorophylls. In: Chemistry and biochemistry of plant pigments (TW Goodwin ed) Acad Press, Londres, 461-488

Juste C, Pouget R (1972) Appréciation du pouvoir chlorosant des sols par un nouvel indice faisant intervenir le calcaire actif et le fer facilement extractible. Application au choix des porte-greffes de la vigne. CR seances Acad Agric Fr 58, 352-357

Juste C, Pouget R (1980) Rôle de certaines caractéristiques du sol sur la sensibilité des plantes à la chlorose. Sci Sol 18, 37-44

Lindsay WL, Norvell WA (1978) Development of a DTPA soil test for zinc, iron, manganese and copper. Soil Sci Soc Am J 42, 421-428

Lopez-Bellido L, Fuentes M (1986) Lupin crop as an alternative source of protein. Adv Agron 40, 239295

Schinas S, Rowell DL (1977) Lime-induced chlorosis. J Soil Sci 28, 351-368

Zelitch I (1971) Photosynthesis, photorespiration and plant productivity. Acad Press, New-York 